Iranian (Iranica) Journal of Energy \& Environment Journal Homepage: www.ijee.net

IJEE an official peer review journal of Babol Noshirvani University of Technology, ISSN:2079-2115

\title{
Anomaly Detection in Smart Homes Using Deep Learning
}

\author{
M. Moallem*, H. Hassanpour, A. Pouyan
}

Faculty of Computer Engineering and Information Technology, Shahrood University of Technology, Shahrood, Iran

\section{PAPER I NFO}

\section{Paper history:}

Received 04 Febraury 2019

Accepted in revised form 24 June 2019

\section{Keywords}

Anomaly Detection

Beam Search

Deep Learning

Long Short Term Memory

Smart Homes

\begin{abstract}
$A B S T R$ R A $C$ T
Smart homes enable many people, especially the elderly and patients, to live alone and maintain their independence and comfort. The realization of this goal depends on monitoring all activities in the house to report any observed anomaly immediately to their relatives or nurses. Anomaly detection in smart homes, just by existing data, is not an easy task. In this work, we train a recurrent network with raw outputs of binary sensors, including motion and door sensors, to predict which sensor will be switched on/off in the next event, and how long this on/off mode will last. Then, using Beam Search, we extend this event into $k$ sequences of consecutive events to determine the possible range of upcoming activities. The error of this prediction i.e., the distance between these possible sequences and the real string of events is evaluated using several innovative methods for measuring the spatio-temporal similarity of the sequences. Modeling this error as a Gaussian distribution allows to assess the likelihood of anomaly scores. The input sequences that are ranked higher than a certain threshold will be considered as abnormal activities. The results of the experiments showed that this method enables the detection of abnormal activities with desirable accuracy.
\end{abstract}

doi: $10.5829 /$ ijee.2019.10.02.10

\section{INTRODUCTION}

The concept of smart homes as a subcategory of pervasive computing has long been the subject of interest in researchers and industrialists. Many researches and applications, such as home automation [1], optimal energy consumption planning [2], provision of telemedicine services [3], and home security and detection of break-ins by intruders [4] have been undertaken in the context of smart homes. However, most of these studies have focused on daily activity recognition and learning the routine of life in a smart home, failing to consider the challenging problem of anomaly detection and the detection of abnormal behaviors.

Anomaly detection and recognizing abnormal behaviors are of paramount importance, specifically in behavior monitoring systems (BMS) that surveil patients and elderly. In BMS any deviation in the daily routine of the subject can be considered as a new complication, an unhealthy habit or trend, and or a change and decrease of the person's abilities [5].

In the literature on machine learning, anomalies are classified into three groups: point anomaly, collective anomaly, and contextual anomaly [6]. Point anomalies usually manifest in the form of the disparity between the value of one or more separate points and other values.
These anomalies in the smart home affect numerical static quantities such as the duration or frequency of doing a task. For example, a short night sleep or frequent visits to the bathroom can be considered as point anomaly. Cumulative anomaly refers to a set of values that are normative on their own but form anomaly when they are put together. The occurrence of this type of anomaly requires some kind of sequential (temporalspatial) or semantic (graph) relationship among the relevant data. For example, leaving TV on, turning on the lights in the bedroom, and sleeping on the bed are not abnormal. However, the consecutive occurrence of these events may suggest that the residents have gone to bed without turning off the TV. Finally, in the contextual anomaly, a single instance of data is considered to be abnormal given its context. This type of anomaly includes several secondary and sometimes unrelated factors and is, therefore, more complex than other types. In smart homes, these factors often embrace spatial and temporal aspects [7, 8]. For example, sleeping in the bedroom is normal and sleeping in the kitchen is abnormal.

This paper is organized as follows: we review the related works in section 2. Section 3 explains the theoretical principles of the research. These principles include the introduction of recurrent networks as well as the explanation and formulation of the problem of smart 
home anomaly detection. Section 4 describes the proposed method and outlines its major points. In section 5 , we introduce a dataset from a real smart home and report the experimental results on this dataset. Finally, conclusions are drawn in section 6 .

\section{LITERATURE REVIEW}

Research on anomaly detection has a long established history. Studies in this area, like many other branches of machine learning, originated from statistics. For example, Grubbs [9] and Enderlein et al. [10] in their seminal old books, have suggested powerful statistical methods for detecting anomalies. Among traditional methods of machine learning, many, including support vector machines [11], neural networks [12], and decision tree [13], have been used to anomaly detection. A crowd of these methods has been proposed by Chandola et al. [6] and Pimentel et al. [14].

In the field of smart homes anomaly detection, the concepts of routine actions and behavior modeling are the key concepts. Most researchers have tried to define and formulate normal behavior based on sensor data in an unsupervised manner. An unsupervised learning technique clusters the sensor data into different categories known as activities and identifys abnormalities as an observation which is deviated from the models of these pre-known activities. To do this, they use one or more of the following methods: logical methods, statistical methods, rule-based methods and machine learning methods.

The stochastic and non-deterministic nature of human activities complicates the modeling of these activities based on the common logic. Some researchers have attempted to take into account this uncertainty by using Bayesian logic and inference. For example, Ordonez et al. [15] defined three probabilistic features: sensor activation likelihood, sensor sequence likelihood, and sensor duration likelihood. Then they used these variables to model the behavior patterns of residents and to identify abnormalities in their behaviors. Using a Markov logic network, Hela et al. [16] have tried to integrate the power of first-order logic with the power of probability and using this probabilistic logic, search for abnormal activities and states in the uncertain space of the data in a smart home.

The hidden Markov model (HMM) is the most frequently used statistical method in processing smart home data. For example, Monekosso et al. [17] modeled sensors outputs sequences using HMM and considered rare observations as anomalies. This researchers did not use any contextual elements such as time or place, but divided a day into time intervals and, by creating a separate model for each interval, enhanced the accuracy of their method in recognizing abnormal activities.
Forkan et al. [18] also used the HMM to detect the anomalies. They used the high-level interpretation of sensor outputs, i.e., activities, as the model input. Also, they measured behavioral changes and health status of individuals respectively by analyzing their daily work and vital signs patterns. The outputs of these three models were given to a fuzzy system to make the final decision about the normality and abnormality of the situation. This complex approach suffers from two major drawbacks: 1) its precision largely depends on the method that is used to detect and extract activities from raw sensor outputs, 2) despite considering the contextual factor of time, it has failed to consider some important periodic variations such as holidays and weather conditions.

Another highly sophisticated method, which is a rulebased method was provided by Yuan et al. [19]. This model, in addition to binary sensors, employed several other sensors such as light, temperature, blood pressure, and sound sensor. The outputs of these sensors have been fused in various ways to form the sequence of activities. In the next step, the proposed system analyses these series using fuzzy logic and case-based reasoning. In this analys is, many contextual variables such as times, places, weekdays, as well as environmental and physiological factors of individuals were taken into account. Nevertheless, the reliance on this method on case-based reasoning means that it requires background knowledge and is not totally data-driven. In fact, Yuan et al. [19] have not provided any model for normal activities. For this reason, some of the cases should be classified into normal and abnormal by experts at the initial stage so that when the system starts to work, the history of activity flow is assessed through this classification, and the knowledge base of the system is updated.

In recent years, the use of machine learning was increased in smart homes studies. Given the variety and spread of human activities, the bulk of these studies have focused on specific types of activities. For example, Novak et al. [20] tried to detect anomalies in everyday activities by employing Self-Organizing Map and found that the duration for these activities is less than 15 minutes. Moshtaghi et al. [21] focused on several temporal and spatial features, but they sufficed to the detection of inertia and inactivity periods, considering these periods as signs of anomalies, especially in the elderly. Paudel et al. [22], were used graphs to analyze and study a series of activities in 400 elderly people to detect abnormalities and symptoms of illness, though all of these people undertook a set of predefined exercises. Several other researchers such as Jakkula [23] have used tools like one class Support Vector Machine (OCSVM) to discover the smart home anomalies. Nonetheless, most of these works have merely focused on point or collective anomalies, failing to consider important contexts such as hours of daytime and night-time, weekdays, seasons, and so on. 
The major weakness of the mentioned researches is that, they have relied on abstract concepts of activity and behavior. These concepts are very diverse for different people and at different times, so it is almost impossible to make a single definition of them. We tried directly use sensor data to detect anomalies, instead of using these high-level concepts. In this way, deep learning helps to extract the main features and frequent pattems automatically. It allows us to implicitly include these concepts in our model.

\section{PRELIMINARIES}

\section{Recurrent neural networks, LSTM, and GRU}

Recurrent Neural Networks are dynamic systems that maintain their internal state during process steps (for example, classification).

This feature is due to the circular communications that exist between adjacent layer neurons. In some models, these connections are seen in the same layer of neurons, or even in the context of a neuron with itself. These recurrent connections allow the network to retain the data of previous steps until the next steps. Thus, recurrent networks have a kind of memory, and therefore their computational model is more powerful than that of the feed forward neural networks [24].

LSTM $^{1}$ networks, instead of neurons use larger and more complex units called memory blocks. The main concept in each memory block is its state, which is a complex and nonlinear outcome of the input of block, output of adjacent blocks, state of the block in previous steps, and the state of the previous block. Each memory block is composed of a memory cell and some gates. The memory cell maintains the current state of the block and the gates handle the inward-outward flow of information. Figure 1 shows the structure of an LSTM memory block. As Figure 1 shows, each gate is a sigmoidal unit whose output ends up to a Hadamard or element-wise product.

In LSTM architecture, gates receive input of the current block $\left(\mathrm{x}_{\mathrm{t}}\right)$ and output of the previous block $\left(\mathrm{h}_{\mathrm{t}-1}\right)$ as inputs, pass them through a sigmoid layer filter, and

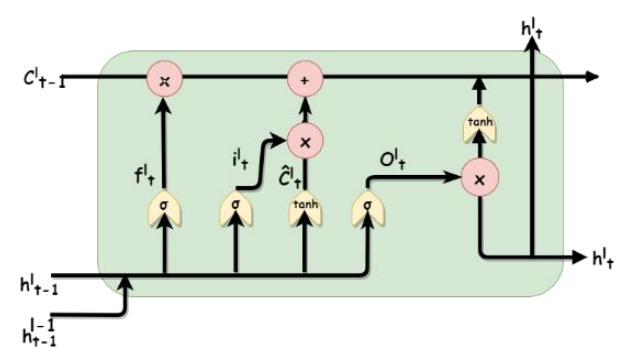

Figure 1. The structure of an LSTM memory block produce a number between 0 and 1 . This output defines the efficacy of the gate. The input gate determines which data should be stored in the memory cell, the forget gate decides how long these data should remain in the block, and the output gate specifies the data that should be mixed in the output of the block. Gers and Schmidhuber [26] added the current state of the block to the each gate's input, named it as peephole connection, and created the most common type of LSTM blocks, known as peephole LSTM. The blocks used in this study are of original type.

An LSTM block can be considered as an F function, which, by receiving the current input $\left(x_{t}\right)$, current state $\left(\mathrm{c}_{\mathrm{t}}\right.$ 1 ), and the output of the previous block $\left(h_{\mathrm{t}-1}\right)$ generates the output of the current block $\left(\mathrm{h}_{\mathrm{t}}\right)$ :

$$
h_{t}=F\left(x_{t}, h_{t-1}, c_{t-1}\right)
$$

The $\mathrm{F}$ function follows the following steps since receives the input until the moment of the generation in the output:

$$
\begin{aligned}
& i_{t}=\sigma\left(W_{x i} * x_{t}+W_{h i} * h_{t-1}+b_{i}\right) \\
& \hat{C}=\tanh \left(W_{x c} * x_{t}+W_{h c} * h_{t-1}+b_{c}\right) \\
& f_{t}=\sigma\left(W_{x f} * x_{t}+W_{h f} * h_{t-1}+b_{f}\right) \\
& C_{t}=f_{t} \odot C_{t-1}+i_{t} \odot \widehat{C_{t}} \\
& o_{t}=\sigma\left(W_{x o} * x_{t}+W_{h o} * h_{t-1}+b_{o}\right) \\
& h_{t}=o_{t} \odot \tanh \left(C_{t}\right)
\end{aligned}
$$

In the above relations, $\mathrm{i}_{\mathrm{t}}, \mathrm{f}_{\mathrm{t}}$, and $\mathrm{o}_{\mathrm{t}}$ represent the input gate, the forget gate, and the output gate, respectively. $W$ and $b$ are parameters of the model, i.e., weights of connections and biases. $\sigma$, Tanh, and $\odot$ are also hyperbolic tangent functions, sigmoid, and the elementwise product operator, respectively.

The GRU ${ }^{2}$ structure proposed in 2014 by Cho et al. [27]

has been derived from the LSTM gated architecture. GRU is simpler than LSTM in at least two aspects: unlike LSTM, GRU does not have any memory cell. Hence, it does not retain the current state, $\mathrm{C}_{\mathrm{t}}$ and transmits it immediately to the next unit after production. Also, in the design of GRU, input gate and forget gate are merged into a new gate called the update gate. Due to these differences, training GRU networks takes less time and data. In contrast, at least theoretically, LSTM networks should be able to learn long term dependencies better than GRU networks. However, there is no rule for selecting between LSTM and GRU. The correct way is built on trial and error and the final choice may depend heavily on the dataset and corresponding task [28].

\footnotetext{
1 Long Short Term Memory

2 Gated Recurrent Unit
} 


\section{Multi-task learning}

There are two methods for simultaneous prediction of two dependent and associated variables. In the first method, we consider them as two independent variables and develop separate models to predict each of them. But, in the second method, the variables simultaneously pass through shared layers, and then each variable is delivered to its specific layers. Each variable has its cost function and the training is done for minimizing an overall cost function that derived from the weighted sum of these functions. This method, called Multitask Learning [29], takes into account the dependence of the relevant variables. Multi-task learning has long been used in machine learning applications, including Natural Language Processing, Speech Recognition and Machine Vision [30]. Application of this method, especially for dependent variables, increases the accuracy of prediction and reduces the risk of overfitting. Figure 2 shows the difference between the usual single-task learning (or single learning) and multi-task learning (or simultaneous learning).

Since we intend to perform a simultaneous prediction of two variables: the next event label and the duration of the event, multi-task learning better suits our purpose and achieves higher performance and accuracy.
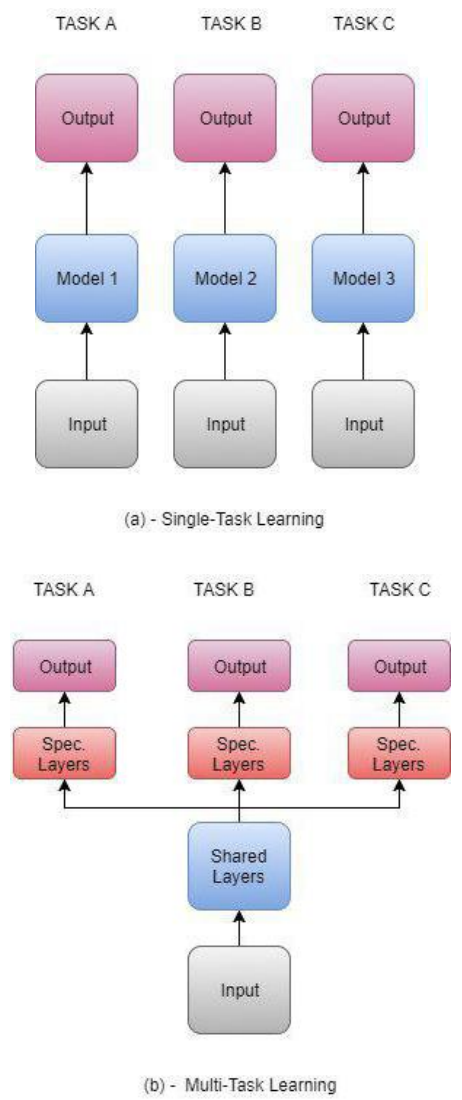

Figure 2. Single-task learning versus multi-task learning.

\section{Anomalies in Smart Homes}

Most of the smart home datasets, record sensor events in the following format:

$$
\mathrm{e}_{\mathrm{i}}=\langle\mathrm{T}, \mathrm{S}, \mathrm{A}\rangle
$$

In this triple, $T$ is timestamp and stands for "sensor activation time", which is often expressed in seconds and includes date too; $S$ stands for "Sensor Identifier" and can contain other information, such as the location or the object where the sensor is attached, and finally, $A$ represents "Sensor Value". Since all of our sensors are binary, this value can be considered as ON or OFF. The index $\boldsymbol{i}$ in the definition of $\boldsymbol{e}_{\boldsymbol{i}}$ denotes that the order of events is important.

However, we convert the sensor event $e_{i}$ into the following triple:

$$
\mathrm{e}_{\mathrm{i}}=\langle\mathrm{T}, \mathrm{SA}, \mathrm{D}\rangle
$$

In this triple, $T$ still stands for timestamp and $S A$ is a string obtained by concatenating $S$ and $A$ values from the previous triple, which is called Event Label. The third element, $D$ or duration, shows the time between the present and previous event. In other words:

$$
\mathrm{D}_{\mathrm{i}}=\mathrm{T}_{\mathrm{i}}-\mathrm{T}_{\mathrm{i}-1}
$$

Naturally, this value is zero at the first event. For example, in our method, the triple $\left\langle\mathrm{T}_{5}, \mathrm{M} 1, \mathrm{ON}\right\rangle$ will be converted into triple $\left\langle\mathrm{T}_{5}, \mathrm{M} 1 \mathrm{ON}, \mathrm{T}_{5}-\mathrm{T}_{4}\right\rangle$.

A series of successive events such as $E$ is called Episode:

$$
\mathrm{E}=\left\langle\mathrm{e}_{1}, \mathrm{e}_{2}, \mathrm{e}_{3}, \ldots ., \mathrm{e}_{\mathrm{n}}\right\rangle
$$

The order of an episode is determined by the activation time of its events (T). An epis ode can often be interpreted as an activity. For instance, if a person leaves the bedroom and go to the kitchen through a hallway and each of these locations has its motion sensor called e1, e2 and e3, then episode $\langle\mathrm{e} 1, \mathrm{e} 2$, e3> will be observed.

We define abnormal behavior as an episode in which:

- has never been observed yet;

- is rarely observed in the past;

- is significantly away from the recent episodes.

The above definitions of anomaly are only related to the events and the order of their occurrence, without considering temporal anomalies. There is anotherkind of anomaly in which:

- an abnormal episode has already been seen, but its temporal coordinates are far different from the previous observations.

For example, several-hours interval between epis odes e2 and $\mathrm{e} 3$ in the above example should be considered abnormal, as it may suggest that someone has suffered an accident before reaching to the kitchen.

\section{THE PROPOSED METHOD}

Figure 3 shows the overall scheme of the proposed method. First, the raw sensors events are preprocessed, 


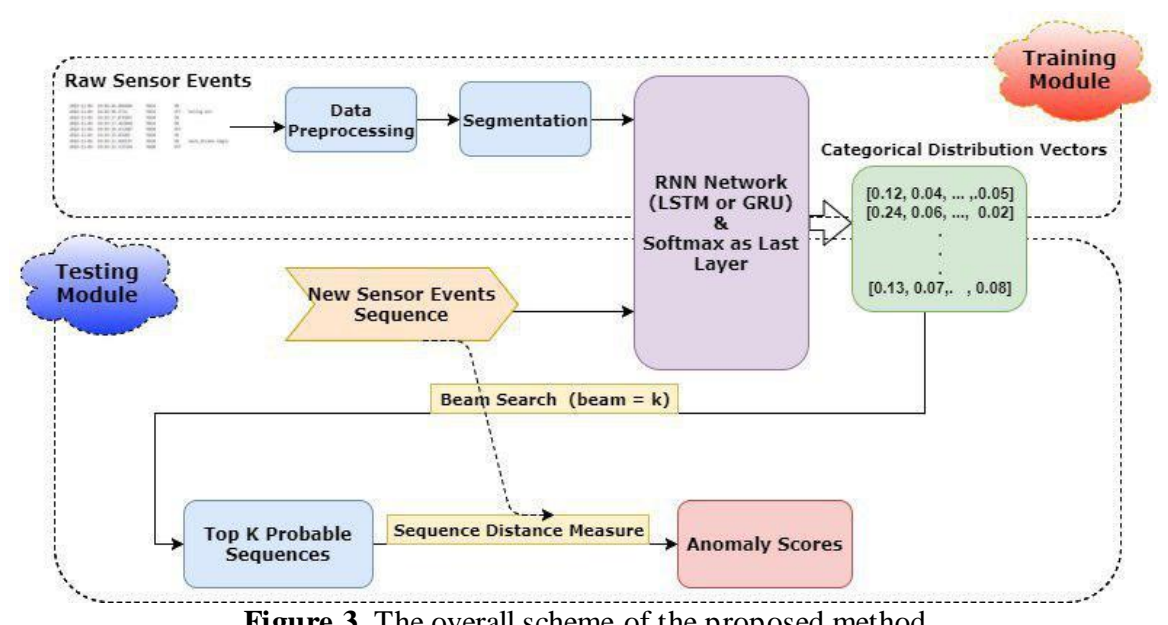

Figure 3. The overall scheme of the proposed method

which includes the following steps:

- By concatenating $\mathrm{S}$ and $\mathrm{A}$ values, SA value is obtained.

- The character string of SA is encoded. This encoding can be carried out by One Hot encoding or Word Embedding method.

- By subtracting the current and the previous event timestamps, D is calculated.

- Timestamp conversion is carried out in a way that periodicity, cycle, and the return of time are considered.

In explaining the last preprocess, it should be noted that applying common ways to express features like weekdays or months could lead to the confusion of classifiers. As an example, using numbers 1 to 7 for weekdays may give the classifier the misperception that $4^{\text {th }}$ and $5^{\text {th }}$ days are more similar than the $1^{\text {st }}$ and $5^{\text {th }}$ days! The same holds true for days of month, even with more complication, as months lengths are different. So, it is generally preferable to use the Fourier transform for expressing the cycling and periodic variables. The above transformation formula, for example for a day's hours, is as follows:

$$
\mathrm{a}=\mathrm{h} \times(2 \pi / 24)
$$

This transformation maps hours into the surface of a circle. Thus, the difference between two hours is expressed as the angle between the two vectors created by this mapping, i.e. ( $\cos (a)$, sin (a)). Figure 4 shows how the days of 3-months, in each of the above methods, is displayed.

In the next step, the input data is segmented into windows of length $n$ and overlapping $m$, to convert the problem into a type of supervised classification. Each window is considered as $\mathrm{X}$ and the next event, i.e., $\mathrm{e}_{\mathrm{n}+1}$, is considered as Y. Now, we must find a mapping that can guess the next event with high precision by receiving an n-events window.

$$
F\left(<e_{i}, e_{i+1}, e_{i+2}, \ldots, e_{i+n}>\right)=e_{i+n+1}
$$

We used LSTM and GRU networks with different structures to find the above mapping. Details of these structures are presented in section 5-2. It should be noted that all utilized networks predict the $S A_{i+n+1}$ and $D_{i+n+}$ 1 components of the next event, and the timestamp of this event, i.e., $T_{i+n+1}$, can be calculated by summing the temporal elements of the previous event, i.e., $T_{i+n}+D_{i+n}$. After training the model by training data, we will have a predictive model that can predict the next event, i.e, $e_{t+1}$, by receiving the input string $\left\langle\mathrm{e}_{1}, \mathrm{e}_{2}, \ldots, \mathrm{e}_{\mathrm{t}}\right\rangle$. In time the detection of point anomalies; but, because we want to find collective and contextual anomalies, this criterion is not applicable to our is sue. In other words, we are looking series applications, the distance between the predicted values and the real values is usually used as a measure for for a sequence of events that their occurrence at a specified time is considered as an anomaly. For this reason, after making the prediction, we add $e_{t}+1$ to the beginning of the input string and remove the its first event (i.e. $e_{1}$ ). Now we have a new $\mathrm{t}$-fold episode, like $<\mathrm{e}_{2}, \mathrm{e}_{3}, \ldots$,

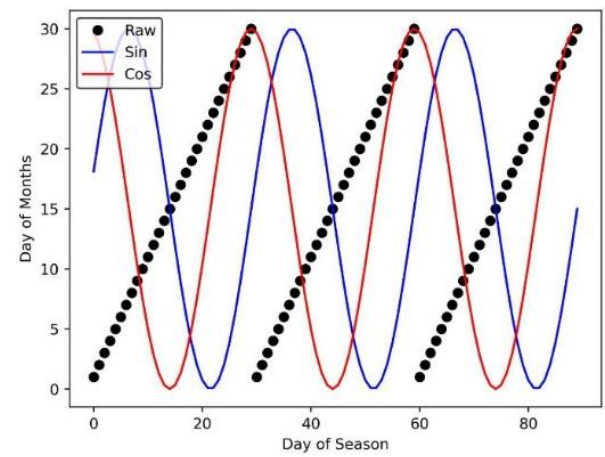

Figure 4. The days of a season with the number of days and the Fourier transform 
$e_{t+1}>$ that can be used to predict the event in the next two steps, $e_{t}+2$. By repeating this process, we bring a sequence of the subsequent probable events. This method, known as the recursive or iterative prediction, is a common practice in the literature on the sequences and time series [31]. The disadvantage of this method is that, despite its high precision in the first steps, its accuracy drops dramatically when the number of steps is increased, due to the accumulation of errors. For this reason, this approach is only suitable for predicting a limited horizon of upcoming events [31].

The distinctive feature of our work is that we use the Beam Search instead of using the usual greedy method to find the next step. Note that the proposed model at each step predicts a single number as duration or $\mathrm{D}$, but the prediction of event label or SA is different. We use a Softmax function to predict this element in the last layer of our networks. The output of this layer is a vector whose length is equal to the number of existing labels and the value assigned to each item is equal to the probability of its occurrence.

At each step, we select the $m$ labels that have the highest probability, and by adding $D$ to them, and computing the associated $T$, we offer $\mathrm{m}$ suggestion for the following event. Then, we make iterative predictions with these $\mathrm{m}$ suggestions and create $\mathrm{m}^{*} \mathrm{~m}$ proposals for the next two steps. By continuing this process in 1 iterations, we will end up having a full and balanced tree, with the depth 1 and $m$ offspring per node. A complete traversing of all paths of this tree, from root to leaves, provides us with $\mathrm{m}^{1}$ probable episodes along with the probability of each node. Now, using the Beam Search method, we can extract some possible sequences of events from these paths. The number of these sequences represents the desired value of $\mathrm{k}$, which is called "beam size."

Beam Search is a heuristic method that explores the tree of the upcoming states and expands only the nodes that are more likely to occur than others [32]. This method is based on Breath-First Search, meaning that it first generates all successors of the states at the current level. The difference here is that the beam search, arranges these nodes based on a target function $\mathrm{f}$, and only stores a predetermined number of best states at each level. This number, known as beam size, is usually determined by temporal and computational - especially memory- limitations. Naturally, larger values will increase the required time and memory for running the program. As the beam-size approaches to infinity, the method gets closer to the Breath-First Algorithm. Beam search is widely used in Text Mining and Natural Language Processing [33].

Now we have $k$ event sequences of length 1 , which are likely to occur based on the available data. The distance between the real episode and this set can be considered as a prediction error and used to compute the Anomaly
Score. These strings contain a set of ordered tuples, in which an element - the event label - is discrete, and the other element -the duration - is continuous. For this reason, none of the methods used to calculate the distance between discrete sequences or time series are applicable to this case.

To calculate this distance, we use the method proposed by Park et al. [34]. In this study, four different similarity functions have been defined. These functions take into account all possible features concerning the differences and similarities of the above tuples. Then the weighted sum of them are used as the final similarity criterion. These functions are expressed in Equations (14) to (18). In these equations, $\mathrm{A}$ and $\mathrm{B}$ refer to the two compared episodes and $n$ denotes their lengths. Also, LCS is the Longest Common Substring of these two episodes, with the length k. Symbols $\|$ and $\{\{\}\}$ are also used to represent the length of a structure (e.g., a sequence) and the length of a MultiSet, respectively. A multiset is a set in which the elements may occur more than once and where several identical members do not reduce to one single member. Finally, $\mathrm{S}$ refers to the final similarity criterion:

$$
\begin{aligned}
& S(A, B)=\sum_{i=1}^{4} w_{i} S_{i} \\
& S_{1}=\frac{\left|L C S_{A, B}\right|}{k} \\
& S_{2}=\frac{\mid\{x \mid x \notin L C S, x \in A \text { or } x \in B\}\} \mid}{n} \\
& S_{3}=1-\frac{S_{t}}{t_{C}} \\
& S_{4}=\frac{\sum_{j=1}^{|L C S|} \mid \frac{\operatorname{Min}\left(d_{j}, d^{\prime}{ }_{j}\right)}{\operatorname{Max}\left(d_{j}, d^{\prime}{ }_{j}\right)}}{k} \\
& \text { In Equation }(17), \text { the function } S_{\mathrm{t}} \text { is defined as: } \\
& S_{t}=\frac{\sum_{j=1}^{|L C S|}\left|t_{j}-t^{\prime}{ }_{j}\right|}{k}
\end{aligned}
$$

It should be noted that among the above criteria, S3 (Equation (17)) and S4 (Equation (18)) contains the concept of time. In these relations, $t j, t^{\prime} j, d j$ and $d^{\prime} j$ represents the timestamp and duration of two events of episodes A and B, which have the same position in the LCS. Finally, $t_{C}$ is the time constant in seconds, which depends on the period under study. For example, to study a 24-h interval, this constant is equal to 86,400 . The value of each sub-function of $\mathrm{Si}$ is normalized to a number within 0 and 1 . Then, the weighted sum of these values is used as the final criterion. By naming this function like $\mathrm{F}$, the difference between the k-member set of the predicted sequences and the real episode will be as follows :

$$
d=\frac{1}{k} \sum_{i=1}^{k} f\left(E, S_{i}\right)
$$

In this equation, $\mathrm{E}$ is the real episode, and $\mathrm{S}_{\mathrm{i}}$ is the $\mathrm{i}$-th predicted sequence. By evaluating the test set with the trained model, an error vector is obtained which will be used to calculate the anomaly score in each input 
window. This vector is modeled as a Gaussian distribution of $\mathrm{N}(\mu, \Sigma)$ [35]. Thus, the probability of observing $\mathrm{p}^{\mathrm{t}}$ error at point $\mathrm{e}^{\mathrm{t}}$ is obtained by calculating the value of $\mathrm{N}$ at the point $\mathrm{e}^{\mathrm{t}}$. We use the Maximum Likelihood Estimation method to calculate $\mu$ and $\Sigma$ values. Now any input window whose $\mathrm{p}^{\mathrm{t}}$ value is greater than the threshold $t$ is considered as an anomaly. The value of this threshold can be determined either statically based on the existing observations or dynamically by maximizing the $\mathrm{F} \beta$ criterion.

\section{EXPERIMENTS}

\section{Dataset}

The dataset used in this study was taken from the Center for Advanced Study of Adaptive Systems (CASAS) located at Washington State University. This center is one of the most prestigious smart home study centers. The contents of the dataset were collected in the course of 220-days - since November 4, 2010, to June 11, 2011 from round-the-clock monitoring of an old woman. To construct this dataset, called Aruba [36], 40 sensors including motion sensors, door closure sensors, and temperature sensors were used. The Aruba dataset contains 11 types of daily activities and 1,719,553 sensor events. Since our study only deals with binary sensors, we eliminated the set of temperature events, reducing the number of these rows to $1,602,818$. An example of the contents of Aruba is shown in Figure 5. This dataset has been primarily designed to recognize and classify daily activities, so there are no distinct and labeled anomalies in it. For this reason, we considered its data as normal, and accordingly, generated 100 sample anomalies manually. These records are in shape of epis ode form and contain one types of anomalies mentioned in section 3-3.

Regretfully, there is currently no public and standard dataset for detecting anomalies in smart homes. For this reason, manual adding of anomalies to public ADL datasets, which were basically designed for activity recognition, is a conventional method utilized by many researchers, including Ye et al. [37], Ordonez et al. [15], and Shin et al. [38].

\section{Implementation}

We conducted a series of experiments to evaluate the proposed method. These experiments differ in criteria such as the length of the input window, the overlapping of these windows, the number of output vectors, and the beam size (the number of output strings of the beam search). Table 1 summarizes the characteristics of these experiments.

We also created various networks as the prediction and anomaly detection model that vary in the type of elements (LSTM or GRU), the number of blocks, the number of layers, the specifications of the fully connected (Dense) layers and Dropout. Table 2 summarizes the characteristics of these networks.

Irrespective of these differences, other learning, and experimental parameters are identical in the experiences. Input data are divided into two sections of 70 and $30 \%$. The first part constitutes the training set and the second part, after the injection of manually generated anomalies, has been used as the test set. The data were delivered to the models in 100-fold batches (batch size $=100$ ), and each network was trained 50 times (epochs $=50$ ). Finally, in all cases, the learning rate was 0.0025 , and the crossentropy function was used as the network loss function. In addition to the above structures, we also used the OneClass Support Vector Machine (OC-SVM). This method has been used in several studies for detecting anomalies in smart homes.

For the comparis on of these methods, we used the Receiver Operating Characteristic (ROC) curve and Area Under the Curve (AUC) [39]. Common measurement criteria, such as precision, accuracy, recall and even the F1 measure, which are frequently used to analyze classification methods, lack the required efficacy and flexibility in detecting anomalies. For this reason, in most of the researches undertaken in this area, ROC and AUC criteria have been adopted [40, 41]. Table 3 shows the AUC values for different experiences. Figure 6 also shows the ROC graph for the same experiences.

These experiments showed that LSTM networks work better. Of course, the training time of LSTM networks is much longer. The superiority of the P2

TABLE 1. Features of experiments

\begin{tabular}{lcccc}
\hline Name & $\begin{array}{c}\text { Length of the } \\
\text { input window }\end{array}$ & $\begin{array}{c}\text { Overlapping } \\
\%\end{array}$ & $\begin{array}{c}\text { Number of } \\
\text { output vectors }\end{array}$ & $\begin{array}{c}\text { Beam } \\
\text { size }\end{array}$ \\
\hline P1 & 4 & 0 & 5 & 6 \\
P2 & 8 & 25 & 8 & 6 \\
P3 & 12 & 50 & 10 & 8 \\
\hline
\end{tabular}

TABLE 2. Features of the applied networks

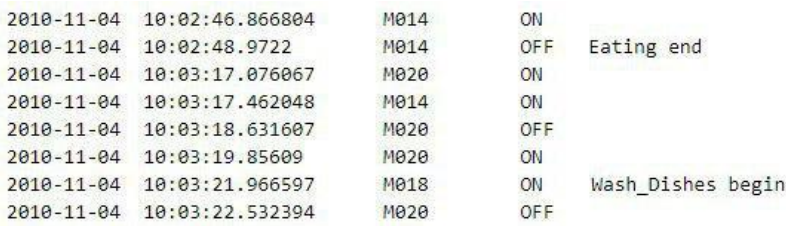

Figure 5. A part of Aruba dataset

\begin{tabular}{lccccc}
\hline Name & Type & $\begin{array}{c}\text { Recurrent } \\
\text { layers }\end{array}$ & $\begin{array}{c}\text { Shared connected } \\
\text { Layers }\end{array}$ & $\begin{array}{c}\text { Dropers } \\
\text { layent }\end{array}$ & Dropout \\
\hline N1 & LSTM & $64-32$ & $1(64)$ & $512-128$ & 0.4 \\
N2 & GRU & $64-32$ & $1(64)$ & $512-128$ & 0.4 \\
N3 & GRU & $64-32$ & 0 & $256-128$ & 0.2 \\
\hline
\end{tabular}


experiment shows that the length of the input window has a significant effect on the improvement of the prediction and the ability to detect abnormalities. In fact, when splitting the input entries into sliding windows, the length of each window should be close to the average length of the user's activity. Also, the overlapping of these windows should be selected so that abnormalities are not considered as normal strings by replication and distribution among adjacent windows. To adjust these parameters, we need to consider the statistics of the data used.

These experiments showed that LSTM networks work better. Of course, the training time of LSTM networks is much longer. The superiority of the P2 experiment shows that the length of the input window has a significant effect on the improvement of the prediction and the ability to detect abnormalities. In fact, when splitting the input entries into sliding windows, the length of each window should be close to the average length of the user's activity. Also, the overlapping of these windows should be selected so that abnormalities are not considered as normal strings by replication and distribution among adjacent windows. To adjust these parameters, we need to consider the statistics of the data used.Increasing the number of output vectors and the

TABLE 3. Features of the applied networks

\begin{tabular}{lc}
\hline Method & AUC value \\
\hline N1P2 & 0.9063 \\
OCSVM & 0.8897 \\
N2P2 & 0.8830 \\
N2P3 & 0.8828 \\
N3P2 & 0.8384 \\
N2P1 & 0.8360 \\
N1P3 & 0.8026 \\
N3P1 & 0.7946 \\
N3P3 & 0.7861 \\
N1P1 & 0.7617 \\
\hline
\end{tabular}

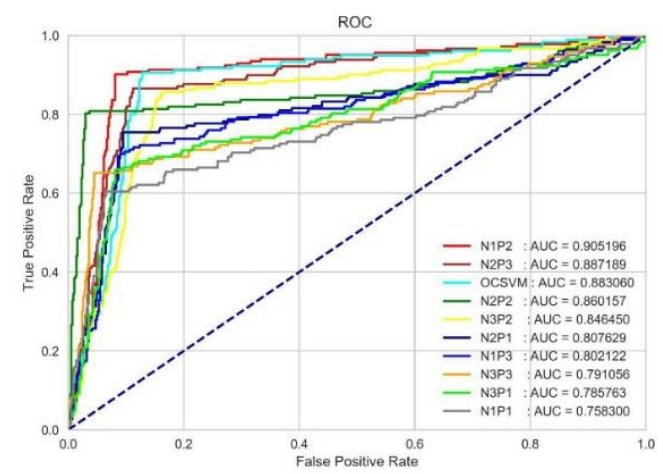

Figure 6. ROC graph for different experiences beam size raise the runtime, but in contrast, it improves the variety and quantity of guesses. As a result, the distance of the proposed set will be reduced to the actual episode. This is probably why the P3 experience has produced good results. We also found that the use of multi-task learning and using shared layers to jointly predicting the next event label and its timestamp increases the accuracy of prediction and the likelihood of anomaly detection. The weakness of the N3 model results is due to the lack of these layers.

All the required programs are written in Python, using Tensorflow and Keras libraries. These programs were run on Linux Mint, relying on the features of the CUDA 8 library to utilize the graphics card capability. The computer was also equipped with an NVidia GeForce 920 MX graphics card with 256 CUDA cores, a quadcore Intel Core i7 $7500 \mathrm{U} 2.7 \mathrm{GHz}$ processorand an 8GB physical memory.

\section{CONCLUSIONS}

In this article, we proposed the use of recurrent networks for anomaly detection in smart homes. Our proposed method does not require any activity recognition mechanism, and the raw output of sensors is received as the inputs. Since the common anomalies in smart homes are of contextual and collective types, we selected some of the most probable upcoming sequences of events using beam search so that their comparison with real episode alerted us about the possible occurrence of the anomalies.

The results of our experiments showed that this method was successful in detecting various anomalies and identifying the range of them with desirable precision. Based on our experience, LSTM networks outperform GRU networks. The truth of the last proposition, and the values of other parameters, such as the size of the input window, their overlapping percentage, the beam size of beam search, among other things, should be examined separately for each dataset.

Along with the strengths of the proposed method, we should point out that the use of deep learning usually requires considerable data, time, and computing resources. This makes our method unsuitable for use as an online tool. An anomaly detection system is expected to act online and promptly detect and report abnormalities in an appropriate time. It should further be noted that common artificial neural networks - Shallow and Deep - could not yet offer insights into uncertainty prediction as it was introduced in this paper. However, finding a way to reduce the temporal cost of the proposed method and transforming it into an online technique for live detection of anomalies are topics that should be further explored for their theoretical and practical values. Besides, using Bayesian neural network (BNN) architecture may improve time series predictions accuracy and uncertainty estimations at scale. 


\section{REFERENCES}

1. Withanage, C., R. Ashok, C. Yuen, and K. Otto, 2014. A comparis on of the popular home automation technologies, In 2014 IEEE Innov. Smart Grid Technol. - Asia (ISGT ASIA), pp: 600-605.

2. Zhou, B., W. Li, K.W. Chan, Y. Cao, Y. Kuang, X. Liu and X. Wang, 2016. Smart home energy management systems: Concept, configurations, and scheduling strategies. Renewable and Sustainable Energy Reviews, 61: 30-40.

3. Majumder, S., E. Aghayi, M. Noferesti, H. Memarzadeh-Tehran, T. Mondal, Z. Pang and M.J. Deen, 2017. Smart Homes for Elderly HealthcareRecent Advances and Research Challenges. Sensors Vol. 17, no. 11 (2017): 2496.

4. Dahmen, J., B.L. Thomas, D.J. Cook and X. Wang, 2017. Activity learning as a foundation for security monitoring in smart homes. Sensors (Switzerland), 17(4): 1-17.

5. Eisa, S. and A. Moreira, 2017. A behaviour monitoring system (BMS) for ambient assisted living. Sensors (Switzerland), Vol. 17(9): (2017): 1946.

6. Chandola, V., A. Banerjee, and V. Kumar, 2009. Anomaly detection. ACM Computing Surveys, 41(3): 1-58.

7. Hayes, M.A. and M.A.M. Capretz, 2014. Contextual anomaly detection in big sensor data. Proceedings 2014 IEEE International Congress on Big Data, BigData Congress 2014, 64-71.

8. Han, J., M. Kamber, and J. Pei, 2011. Data Mining: Concepts and Techniques. 3rd ed. Morgan Kaufmann Publishers Inc.

9. Grubbs, F.E., 1969. Procedures for Detecting Outlying Observations in Samples. Technometrics, 11(1): 1-21.

10. Enderlein, G., 1987. Hawkins, D. M.: Identification of Outliers. Chapman and Hall, London - New York 1980, 188 S., £ 14, 50. Biometrical Journal, 29(2): 198.

11. Ma, J. and S. Perkins, 2003. Time-series novelty detection using one-class support vector machines, In Proc. Int. Jt. Conf. Neural Networks, 2003., IEEE, pp: 1741-1745.

12. Tavares Ferreira, E.W., G. Arantes Carrijo, R. de Oliveira and N. Virgilio de Souza Araujo, 2011. Intrusion Detection System with Wavelet and Neural Artifical Network Approach for Networks Computers. IEEE Latin America Transactions, 9(5): 832-837.

13. Depren, O., M. Topallar, E. Anarim, and M.K. Ciliz, 2005. An intelligent intrusion detection system (IDS) for anomaly and misuse detection in computer networks. Expert systems with Applications Vol. 29, no. 4 (2005): 713-722.

14. Pimentel, M.A.F., D.A. Clifton, L. Clifton, and L. Tarassenko, 2014. A review of novelty detection. Signal Processing, 99: 215-249.

15. Ordonez, F.J., P. de Toledo and A. Sanchis, 2015. Sensor-based Bayesian detection of anomalous living patterns in a home setting. Personal and Ubiquitous Computing, 19(2): 259-270.

16. Hela, S., B. Amel, and R. Badran, 2018. Early anomaly detection in smart home: A causal association rule-based approach. Artificial Intelligence in Medicine, Vol. 91 (2018) 57-71.

17. Monekosso, D.N. and P. Remagnino, 2010. Behavior analysis for assisted living. IEEE Transactions on Automation Science and Engineering, 7(4): 879-886.

18. Forkan, A.R.M., I. Khalil, Z. Tari, S. Foufou and A. Bouras, 2015. A context-aware approach for longterm behavioural change detection and abnormality prediction in ambient assisted living. Pattern Recognition, 48(3): 628-641.

19. Yuan, B. and J. Herbert, 2014. Context-aware Hybrid Reasoning Framework for Pervasive Healthcare. Personal Ubiquitous Comput., 18(4): 865-881.

20. Novak, M., M. Binas and F. Jakab, 2012. Unobtrusive anomaly detection in presence of elderly in a smart-home environment. Proceedings of 9th International Conference, ELEKTRO 2012, (June 2016): 341-344.

21. Moshtaghi, M., I. Zukerman, and R. Andrew Russell, 2015. Statistical models for unobtrusively detecting abnormal periods of inactivity in older adults. User Modeling and User-Adapted Interaction Vol. 25, no. 3 (2015): 231-265.

22. Paudel, R., W. Eberle, and L.B. Holder, 2018. Anomaly Detection of Elderly Patient Activities in Smart Homes using a Graph-Based Anomaly Detection of Elderly Patient Activities in Smart Homes using a Graph-Based Approach. (July): https://scholar.google.com/citations?user=v_Lx81Y AAAAJ\&hl=en\&oi=sra

23. Jakkula, V. and D.J. Cook, 2011. Detecting Anomalous Sensor Events in Smart Home Data for Enhancing the Living Experience. AAAI Workshop on Artificial Intelligence and Smarter Living The Conquest of Complexity, (June 2015): 33-37.

24. Lipton, Z.C., J. Berkowitz and C. Elkan, 2015. A Critical Review of Recurrent Neural Networks for Sequence Learning. 1-38. arXiv:1506.00019v4

25. Hochreiter, S. and J. Urgen Schmidhuber, 1997. Long Short-Term Memory. Neural Computation, 9(8): 1735-1780. 
26. Gers, F.A. and J. Schmidhuber, 2000. Recurrent nets that time and count, In Proc. IEEE-INNS-ENNS Int. Jt. Conf. Neural Networks. IJCNN 2000. Neural Comput. New Challenges Perspect. New Millenn., IEEE, pp: 189-194 vol.3.

27. Cho, K., B. van Merriënboer, C. Gulcehre, F. Bougares, H. Schwenk and Y. Bengio, 2014. Learning Phrase Representations using RNN Encoder-Decoder for Statistical Machine Translation. arXiv preprint arXiv:1406.1078 (2014).

28. Chung, J., C. Gulcehre, K. Cho and Y. Bengio, 2014. Empirical evaluation of gated recurrent neural networks on sequence modeling, In NIPS 2014 Work. Deep Learn. December 2014,

29. Caruana, R., 1997. Multitask Learning. Machine Learning, 28(1): 41-75.

30. Collobert, R., R. Collobert and J. Weston, 2008. A unified architecture for natural language processing: Deep neural networks with multitask learning. 160167.

31. Kline, D., 2004. Methods for Multi-Step Time Series Forecasting with Neural Networks. In Neural networks in business forecasting, pp. 226-250. IGI Global, 2004.

32. Reddy, D.R.( D. of C.S., 1977. Speech Understanding Systems: A Summary of Results of the Five-Year Research Effort at carnegie-mellon university." Internal Document. August (1977).

33. Freitag, M., and Y. Al-Onaizan, 2017. Beam Search Strategies for Neural Machine Translation. arXiv preprint arXiv:1702.01806 (2017).

34. Park, K., Y. Lin, V. Metsis, Z. Le and F. Makedon,
2010. Abnormal human behavioral pattern detection in assisted living environments. Proceedings of the 3rd International Conference on PErvasive Technologies Related to Assistive Environments - PETRA '10.

35. Malhotra, P., L. Vig, G. Shroff, and P. Agarwal, Long Short Term Memory Networks for Anomaly Detection in Time Series. In Proceedings, p. 89. Presses universitaires de Louvain, 2015

36. Cook, D., 2010. Learning Setting-Generalized Activity Models for Smart Spaces. IEEE intelligent systems no. 99 (2010): 1.

37. Ye, J., G. Stevenson and S. Dobson, 2016. Detecting abnormal events on binary sensors in smart home environments. Pervasive and Mobile Computing, 33: $32-49$.

38. Shin, J.H., B. Lee and K.S. Park, 2011. Detection of abnormal living patterns for elderly living alone using support vector data description. IEEE Transactions on Information Technology in Biomedicine: A Publication of the IEEE Engineering in Medicine and Biology Society, 15(3): 438-448.

39. Fawcett, T., 2006. An introduction to ROC analysis. Pattern Recognition Letters, 27(8): 861-874.

40. Goldstein, M., M. Goldstein, and S. Uchida, 2016. A Comparative Evaluation of Unsupervised Anomaly Detection Algorithms for Multivariate Data. PLoS ONE, (April): 1-31.

41. Emmott, A., S. Das, T. Dietterich, A. Fern and W.K. Wong, 2015. A Meta-Analysis of the Anomaly Detection Problem. arXiv preprint arXiv:1503.01158 (2015).

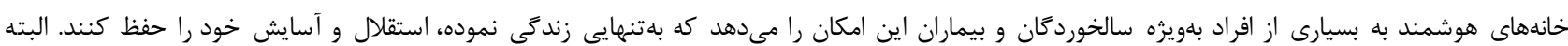

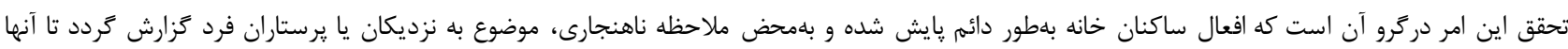

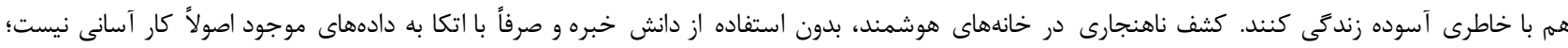

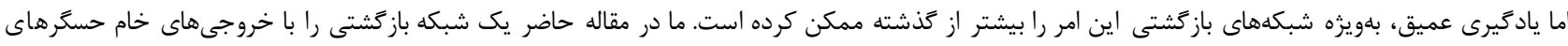

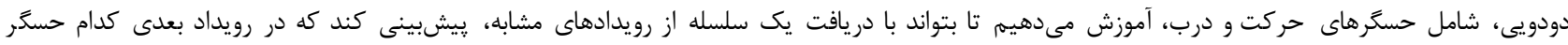

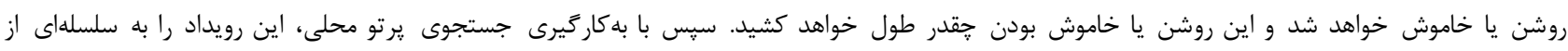

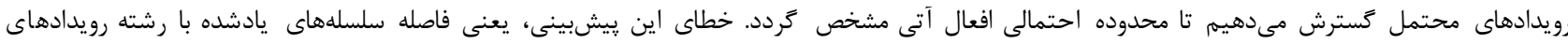

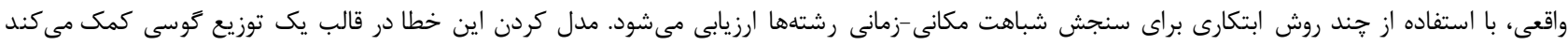

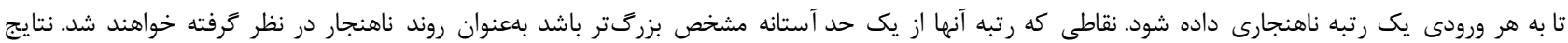

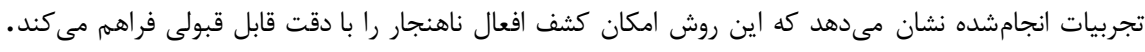

